\title{
Summertime blues
}

\author{
Scientific journals are entirely dependent on the multitude of researchers prepared to spend precious time on peer \\ review. Are we asking too much, especially when there is so much else they could be doing?
}

T he summer months in the Northern hemisphere, June to August specifically, can be a tough time for journal editors. Mary Williams, Features Editor of The Plant Cell, summed it up very well in a tweet on 20 August:

“A special 'thank you' to everyone who is accepting reviewer requests just now. August is the second most difficult month (just behind December) for finding people willing to review manuscripts. And because everyone wants to submit before their holidays, we are flooded!"

The inter-year break in the academic calendar not only provides researchers with the opportunity to take well-earned vacations, but also makes for a very attractive time to hold conferences. What is true for researchers is also true for editors. We also want to find out about the latest goings-on in our field, meet up with old acquaintances and make new ones. One of our editorial colleagues, who handles plant science for another journal (which shall remain nameless), attended five conferences back-to-back through June and July of this year, while the last time that all of the Nature Plants editors were at their respective desks was sometime in June.

We certainly echo Mary's sincere thanks to those researchers who, despite the many other things they could be doing, still agree to provide us with the advice and opinions without which we could not do our job. However, Jennifer Rohn of University College London, UK, recently made a very persuasive case that researchers need to shut off for at least some of their summer break (Nature 572, 417; 2019). Once there was a time when the refereeing load of academics was at a level that could be easily managed, making an "unspoken pact of reciprocity" within the academic community sufficient to provide "a moral obligation to peer review", even during time off. However, "my decade at the university has seen a steep rise in teaching load, mentoring duties and mindless administrative tasks", says Rohn, "and don't get me started on committee meetings". Even the minimum level of academic chores needed to keep her laboratory ticking over means that "most days, my son asks, 'Mama, are you working again?"”

We understand this very well and are constantly amazed by the time and knowledge given to us by our referees. We are acutely aware that the compensations for reviewing are indirect at best, and so wholly unquantifiable that it is almost impossible for any reviewer to answer the question is it worth it?', let alone the more nuanced economic question of 'how much reviewing should I do to maximize my rate of return?'

There are selfish benefits to be had from reviewing. For example, reviewers get to see papers before publication, giving them a time advantage over competitors who have to wait for formal publication. However, for most people the primary reason for reviewing is altruism. The scientific endeavour is so reliant on the process of peer review of both completed research and grant applications, that to not act as a reviewer is to welch on Rohn's "pact of reciprocity".

Given our fundamental dependence on persuading already overworked researchers to act as reviewers, what can journals and their editors do to at least acknowledge this debt without disrupting our attempts to maintain fairness, impartiality and an absence of bias in the peer review process? Publishing the names of reviewers is one small thing that can be done to publicly acknowledge our debt. There is an obvious concern that this may interfere with the anonymity of the process, therefore many journals publish lists of their reviewers on a yearly basis. This is impersonal and it is difficult to imagine anyone scouring through such lists containing hundreds of names to see who has, and has not, been involved in a submission in the previous year.

Alternatively, reviewers can be acknowledged on individual studies. Nature has been doing this for several years, and here at Nature Plants, we are currently running a trial to see whether this will work for us. In the 'Additional Information' section of published papers, you will find a statement from us thanking the reviewers for "their contribution to the peer review of this work". This does reveal the identity of the reviewers (although not unequivocally whether they were the legendarily ferocious reviewer three), and so we ask reviewers first whether they are happy for their names to be released, and if not, we will thank them as 'other anonymous reviewers'. So far, most reviewers seem happy to be named once a paper is published, but it is early days for the trial and this only acknowledges reviewers of ultimately published papers.

More concrete rewards for reviewers do exist. Some journals have been said to send a bottle of wine to all its reviewers at the end of each year, making no distinction as to how many reviews had been performed by each individual or how useful they have been. Some journals offer their most active reviewers a free subscription to the journal, some making this transferable to help support researchers where access to the literature is limited. At one time, the openaccess journal PeerJ offered a reduction on its article processing charge (APC) so that, after reviewing a relatively small number of papers, a reviewer could publish their next paper for free. There is also the perennial suggestion that reviewers should be paid for their time, which opens up a debate about what a minimal level of service a reviewer would need to deliver, in terms of speed and quality of review, to receive payment; and that is before considering whether the introduction of a further potential source of conflicts of interest into a system already rife with opportunities for bias, favouritism and self-interest, is advisable.

As we put the 'second most difficult month' for finding reviewers behind us for another year, we just want to thank everyone who has advised us and everyone who will in the future. Moreover, we want to thank everyone who we have asked and who has had to decline - we never doubt the multifarious calls on your time, or your need for time away from the laboratory bench and office desk. Above all, we hope that whatever you have done in the last three months, it has been enjoyable, profitable and relaxing in equal measure.

Published online: 10 September 2019 https://doi.org/10.1038/s41477-019-0523-8 